P09.05 PLASMA CD27, A SURROGATE OF INTRATUMORAL CD27-CD70 INTERACTION, CORRELATES WITH IMMUNOTHERAPY RESISTANCE IN RENAL CANCER

\begin{abstract}
1,2N Benhamouda, 'I Sam*, ${ }^{1} \mathrm{~N}$ Epaillard, ${ }^{1,2} \mathrm{~A}$ Gey, 1,2A Saldmann, 1,2 Jineau, ${ }^{3} \mathrm{M}$ Hasan, ${ }^{4} \mathrm{~V}$ Verkarre, ${ }^{3} \mathrm{~V}$ Libri, ${ }^{3,5} \mathrm{~S}$ Mella, ${ }^{1,2} \mathrm{C}$ Granier, ${ }^{4} \mathrm{C}$ Broudin, ${ }^{5} \mathrm{P}$ Ravel, ${ }^{3,5} \mathrm{~B}$ Jabla, ${ }^{6} \mathrm{~N}$ Chaput, ${ }^{7} \mathrm{~L}$ Albiges, ${ }^{8,9} \mathrm{Y}$ Vano, ${ }^{10} \mathrm{O}$ Adotevi, ${ }^{1,8} \mathrm{~S}$ Oudard, ${ }^{1,2} \mathrm{E}$ Tartour. ${ }^{1}$ INSERM U970 PARCC Université de Paris, Paris, France; ${ }^{2}$ Service d'Immunologie Biologique, APHP, Hôpital Européen Georges Pompidou (HEGP), Paris, France; ${ }^{3}$ Cytometry and Biomarkers UTechS, Center for Translational Science, Institut Pasteur, Paris, France; ${ }^{4}$ Department of Pathology, APHP, HEGP, Paris, France; ${ }^{5}$ IRCM - INSERM U1194, Institut de Recherche en Cancérologie de Montpellier, Montpellier, France; ${ }^{6}$ Université Paris-Saclay. Laboratory of Immunomonitoring in oncology, Institut Gustave Roussy, Paris, France; ' Université ParisSaclay. Department of Medical Oncology. Institut Gustave Roussy, Paris, France; ${ }^{8}$ Department of Medical Oncology, HEGP, Paris, France; ${ }^{9}$ Centre de Recherche des Cordeliers, INSERM UMRS1138, Université de Paris, Paris, France; ${ }^{10}$ University Bourgogne Franche-Comté, INSERM, EFS BFC, UMR1098; INSERM CIC1431, Department of Pneumology, University Hospital of Besançon, Besançon, France
\end{abstract}

\subsection{6/jitc-2021-ITOC8.55}

Background CD70, a costimulatory molecule on antigen presenting cells, is known to activate CD27-expressing $\mathrm{T}$ cells. CD27-CD70 interaction leads to the release of soluble CD27 (sCD27). However, persistent interaction of CD27 and CD70 such as in chronic infection may exhaust the $\mathrm{T}$ cell pool and promote apoptosis. Surprisingly, our analysis based on TCGA database show that clear cell renal cell carcinoma (ccRCC) expresses the highest levels of CD70 among all solid tumors. Despite the important clinical efficacy of immunotherapy by anti-PD-1 in RCC patients, the overall response to anti-PD1 remains modest. The relationship between the CD27-CD70 interaction in the RCC and the response to immunotherapy is still unclear.

Materials and Methods To study the CD27 and CD70 expression in the tumor microenvironment (TME), FFPE tumor tissues from 25 RCC patients were analysed using multiplex in situ immunofluorescence. 10 fresh RCC tumor samples were collected to analyse the phenotype of $\mathrm{CD} 27^{+} \mathrm{T}$ cells by flow cytometry and 4 samples were proceeded for single-cell RNAseq analysis. A cohort of metastatic RCC patients $(n=35)$ treated by anti-PD-1 were enrolled for the measurement of plasma sCD27 by ELISA and the survival analysis is also realized.

Results In the TME, we demonstrated that $\mathrm{CD} 27^{+} \mathrm{T}$ cells interact with CD70-expressing tumor cells. In fresh tumors from RCC patients, CD $27^{+} \mathrm{T}$ cells express higher levels of cleaved caspase 3 (a classical marker of apoptosis) than CD27 $\mathrm{T}$ cells. We confirmed the apoptotic signature (BAX, FASLG, BCL2L11, CYCS, FBXO32, LGALS1, PIK3R1, TERF1, TXNIP, CDKN2A) of $\mathrm{CD}_{2} 7^{+} \mathrm{T}$ cells by single-cell RNAseq analysis. $\mathrm{CD} 27^{+} \mathrm{T}$ cells also had a tissue resident memory $\mathrm{T}$ cell phenotype with enriched gene expression of ITGAE, PRDM1, RBPJ and ZNF683. Moreover, CD27 ${ }^{+} \mathrm{T}$ cells display an exhaustion phenotype with the expression of multiple inhibitory receptors gene signature (PDCD1, CTLA4, HAVCR2, LAG3, etc). Besides, intratumoral CD27-CD70 interaction significantly correlates with plasma sCD27 concentration in RCC $(p=0.0017)$. In metastatic RCC patients treated with anti-PD-1, higher levels of $\mathrm{sCD} 27$ predict poor overall survival $(\mathrm{p}=0.037)$, while it did not correlate with inflammatory markers or clinical prognostic criteria.

Conclusions In conclusion, we demonstrated that sCD27, a surrogate of $\mathrm{T}$ cell dysfunction in tumors likely induced by persistent interactions of $\mathrm{CD} 27^{+} \mathrm{T}$ cells and CD70-expressing tumor cells, is a predictive biomarker of resistance to immunotherapy in mRCC. To our knowledge, this is the first report showing that a peripheral blood biomarker may reflect certain aspects of the tumor-host interaction in the tumor microenvironment. Given the frequent expression of CD70 and CD27 in solid tumors, our findings may be further extended to other types of tumors. CD70-CD27 interaction could thus be considered as a mechanism of tumor escape, but also a novel therapeutic target in cancers.

Disclosure Information N. Benhamouda: None. I. Sam: None. N. Epaillard: None. A. Gey: None. A. Saldmann: None. J. Pineau: None. M. Hasan: None. V. Verkarre: None. V. Libri: None. S. Mella: None. C. Granier: None. C. Broudin: None. P. Ravel: None. B. Jabla: None. N. Chaput: None. L. Albiges: None. Y. Vano: None. O. Adotevi: None. S. Oudard: B. Research Grant (principal investigator, collaborator or consultant and pending grants as well as grants already received); Modest; SIRIC CARPEM, FONCER. E. Tartour: B. Research Grant (principal investigator, collaborator or consultant and pending grants as well as grants already received); Modest; Fondation ARC, INCA PLBio, Labex Immuno-Oncology, SIRIC CARPEM, FONCER, IDEX université de Paris, Inserm Transfert.

\section{P09.06 INVESTIGATING VARIOUS PATIENT PARAMETERS AS PROGNOSTIC MARKERS FOR PATIENTS WITH ADVANCE STAGE NASOPHARYNGEAL CARCINOMA UNDERGOING INDUCTION CHEMOTHERAPY FOLLOWED BY EPSTEIN- BARR VIRUS CYTOTOXIC T-LYMPHOCYTE IMMUNOTHERAPY}

${ }^{1} \mathrm{~A}$ Chu ${ }^{*},{ }^{2} \mathrm{~S}$ Han, ${ }^{2} \mathrm{H}$ Toh. 'Duke-NUS Medical School, Singapore, ${ }^{2}$ National Cancer Centre, Singapore

\subsection{6/jitc-2021-ITOC8.56}

Background Previous prospective phase II study conducted by our research group at the National Cancer Centre Singapore had shown the efficacy of combined induction chemotherapy followed by cytotoxic T-lymphocyte (CTL) immunotherapy as a first-line treatment for advance nasopharyngeal carcinoma (NPC) - i.e. median survival for patients treated with combined therapy was 29.9 months, compared to 17.7 months for patients who received only standard chemotherapy. ${ }^{1}$ Using the same data set, we further investigate the correlation between various patient factors (Eastern Cooperative Oncology Group (ECOG) score, gender, age, initial stage of cancer, neutrophil-to-lymphocyte ratio (NLR), initial EBV-DNA titre) on overall survival (OS). This is to further validate our hypothesis that the improved OS is due to an effect of treatment and not due to intrinsic patient factors.

Materials and Methods Survival distribution curves were estimated using the Kaplan-Meier method and differences were compared statistically using log-rank test. IBM SPSS statistics software package (v. 22) was used for the purpose of statistical analysis. Overall survival was defined as time from diagnosis to date of event (date of death/date of last follow-up). For analysis of overall survival, data for patients who were alive or who were lost to follow-up were censored at the end of study period.

Results It was revealed that lower ECOG score, a scale used to assess the physical condition of patients, correlated with longer OS while other characteristics such as gender, age, initial stage of cancer, NLR, and initial EBV-DNA titre did not 